\title{
TWO EXTENDING CRACK PROBLEMS IN LINEAR VISCOELASTICITY THEORY*
}

\author{
BY
}

G. A. C. GRAHAM

Simon Fraser University

1. Introduction. The analysis of the growth of cracks is one of those areas of linear viscoelasticity theory which presents difficulties not arising in classical elasticity theory. The first of these difficulties is to solve viscoelastic boundary value problems that involve time-dependent boundary regions. This variation of the boundary regions precludes the application of integral transforms (with respect to time) to the solution of the problems and so excludes the classical correspondence principle (see [1]) as a possible method of solution. In the second place, if it is an energy criterion for crack extension that we are looking for, there is the problem of taking care of the energy dissipation which takes place in viscoelastic bodies but is absent in the elastic theory.

Previously Williams and others [2] have used the elastic solution for the distribution of stress in the neighborhood of the tip of a crack together with critical strain hypotheses to investigate crack growth. Energy criteria which take account of dissipation have also been used by Williams [3] but only for simplified geometries like growing spherical holes. More recently Willis [4] has exploited a Baranblatt type of fracture criterion in investigating a steady-state dynamic viscoelastic crack propagation problem.

In this paper, using the quasi-static theory as a point of departure, formulae are derived for the distribution of stress and displacement obtaining in an infinite linear viscoelastic body which contains an extending plane crack. Both the symmetrically loaded two-dimensional and the axisymmetrically loaded three-dimensional cases are treated. In either case the problem may be reduced to that of finding the displacement and stress fields set up in a viscoelastic half-space by the action of a distributed normal pressure acting over a time-dependent part of its surface, which corresponds to the area occupied by the crack. Outside this area the normal surface displacement is required to vanish, while the shear traction is required to vanish at all points of the surface. This therefore gives rise to mixed boundary value problems for which the regions, over which the different types of boundary condition prescribed vary with time and therefore the correspondence principle is not applicable.

We have used an extension of the correspondence principle given in [5] which is applicable to both the problems under consideration. Formulas for (i) the stress distribution in the plane of the crack, (ii) the displacement over the surface of the crack and (iii) the stress intensity factor are given for both cases. The particular circumstance that the applied internal pressure is the same at all points of the surface of the crack is investigated and, for the two-dimensional problem, the effect of viscoelasticity on the displacement over the crack surface is demonstrated.

* Received November 7, 1968. Part of this work was sponsored by the U. S. Joint Services Advisory Group under Contract No. AF-AFOSR-444-66 with the Applied Mathematics Research Group, North Carolina State University, Raleigh, and the rest by Grant No. A4831 of the National Research Council of Canada. 
Finally, with the aid of previous considerations, the effect of stress-free extending cracks in viscoelastic bodies which are subject to time-dependent tensile stress at infinity is considered. The rate of decline in the potential energy of the body, due to a smooth virtual extension of the crack, is calculated. Following Griffith [6] we compare this with a quantity which is proportional to a characteristic energy of tearing of the material, and obtain conditions for the growth of such cracks. These conditions take the same form as the conditions for the rupture of elastic bodies that are associated with the names of A. A. Griffith and R. A. Sack (see Sneddon [7]).

2. Field equations of quasi-static linear viscoelasticity theory for homogeneous and isotropic bodies. Consider a fixed region $R$ occupied by a linear viscoelastic material. Let $u_{i}(x, t), \epsilon_{i j}(x, t)$ and $\sigma_{i j}(x, t)$ denote the Cartesian components of displacement, strain and stress respectively, which are defined for all $(x, t)$ on $R \times[0, \infty)$. In Cartesian tensor notation the relevant fundamental system of field equations appropriate to the linear quasi static theory of viscoelasticity, for homogeneous and isotropic bodies, and for zero body force, may be written as follows:

$$
\begin{aligned}
2 \epsilon_{i j}(x, t) & =u_{i, j}(x, t)+u_{i, i}(x, t), \\
\sigma_{i i, i}(x, t) & =0, \quad \sigma_{i j}(x, t)=\sigma_{i i}(x, t), \\
\sigma_{i i}(x, t) & =\left[\epsilon_{i j} * d G_{1}+\frac{1}{3} \delta_{i j} \epsilon_{k k} * d\left(G_{2}-G_{1}\right)\right](x, t) .
\end{aligned}
$$

Here $G_{1}(t)$ and $G_{2}(t)$, which are assumed to be independent of $x$, are the relaxation moduli in shear and isotropic compression respectively. Moreover we have used the notation that if $f$ and $g$ are functions of position and time then $f * d g$ stands for the function defined by

$$
[f * d g](x, t)=g(x, 0) f(x, t)+\int_{0}^{t} f(x, t-\theta) g^{\prime}(x, \theta) d \theta,
$$

where the dash indicates that we take the time derivative of the function indicated, so that

$$
g^{\prime}(x, \theta)=\partial g(x, \theta) / \partial \theta
$$

If $f(x, 0) \neq 0$ then there is a unique function, denoted by $f^{-1}(x, t)$, for which

$$
\left[f * d f^{-1}\right](x, t)=\left[f^{-1} * d f\right](x, t)=1 .
$$

We shall use the notation

$$
f^{T}(x, s)=\mathscr{L}\{f(x, t) ; t \rightarrow s\}=\int_{0}^{\infty} f(x, t) e^{-s t} d t
$$

for the Laplace transform with respect to time of a function $f(x, t)$. Applying the Laplace transform to Eqs. (1)-(3) and (6), we find that

$$
\begin{aligned}
2 \epsilon_{i j}^{T}(x, s) & =u_{i, j}^{T}(x, s)+u_{i, i}^{T}(x, s), \\
\sigma_{i i, i}^{T}(x, s) & =0, \quad \sigma_{i j}^{T}(x, s)=\sigma_{j i}^{T}(x, s), \\
\sigma_{i i}^{T}(x, s) & =s G_{1}^{T}(s) \epsilon_{i i}^{T}(x, s)+\frac{1}{3} \delta_{i j} s\left(G_{2}-G_{1}\right)^{T}(s) \epsilon_{k k}^{T}(x, s),
\end{aligned}
$$

and

$$
f^{T}(x, s) f^{-1 T}(x, s)=1 / s^{2}
$$


We note here also that the fundamental system of field equations governing the linear theory of elastostatics for homogeneous and isotropic bodies and for zero body force are (1), (2) together with

$$
\sigma_{i i}(x, t)=2 \mu \epsilon_{i j}(x, t)+\frac{2 \mu \nu}{1-2 \nu} \delta_{i i} \epsilon_{k k}(x, t),
$$

where $\mu$ denotes the shear modulus and $\nu$ Poisson's ratio for the material. The correspondence principle [1] relates solutions of the system of Eqs. (8)-(10) with solutions of the Laplace transforms of the system (1), (2), (12).

3. A two-dimensional extending crack problem. Consider an infinite linear viscoelastic medium containing a plane crack $|x| \leq a(t)$, in the plane $z=0$, which is opened by a symmetrical normal pressure acting on its surface. The plane displacement stress field is the same as that obtaining in a semi-infinite body $z \geq 0$ when its surface is subjected to the boundary conditions ${ }^{1}$

$$
\begin{aligned}
& \sigma_{z x}(x, 0, t)=0, \quad-\infty<x<\infty, \\
& \sigma_{z z}(x, 0, t)=-p(|x|, t), \quad|x| \leq a(t), \\
& u_{z}(x, 0, t)=0, \quad|x|>a(t),
\end{aligned}
$$

and the conditions at infinity

$$
\sigma_{s z}(x, z, t) \rightarrow 0, \sigma_{x x}(x, z, t) \rightarrow 0, \sigma_{z x}(x, z, t) \rightarrow 0, \text { as }\left(x^{2}+z^{2}\right) \rightarrow \infty .
$$

Except when $a(t)$ remains constant with time, the classical correspondence principle of viscoelasticity theory is not applicable to the solution of the problem determined by equartions (1)-(3) and the conditions (13), (14). However, if we denote by the superscript $e$ the one-parameter family of solutions, to the plane strain problem, determined by the conditions (13), (14) and the field equations (1), (2) and (12) we have in particular that (see Sneddon [8])

$$
\begin{array}{cl}
\sigma_{z z}^{*}(x, 0, t) & =\left(\frac{2}{\pi}\right)^{1 / 2}\left\{\frac{|x| g(a(t), t)}{\left[x^{2}-a^{2}(t)\right]^{1 / 2}}-g(0, t)-\int_{0}^{a(t)} \frac{|x| d g(v, t) / d v}{\left[x^{2}-v^{2}\right]^{1 / 2}} d v\right\}, \quad|x|>\begin{array}{r}
a(t) \\
u_{z}^{*}(x, 0, t)
\end{array}=\frac{1-\nu}{\mu}\left(\frac{2}{\pi}\right)^{1 / 2} \int_{|x|}^{a(t)} \frac{v g(v, t)}{\left[v^{2}-x^{2}\right]^{1 / 2}} d v, \quad|x| \leq a(t)
\end{array}
$$

where

$$
g(v, t)=\left(\frac{2}{\pi}\right)^{1 / 2} \int_{0}^{v} \frac{p(|x|, t) d x}{\left[v^{2}-x^{2}\right]^{1 / 2}} .
$$

Since the elastic constants are absent from (15) and appear as a separate factor in (16) we see that the extension of the correspondence principle given in [5] is applicable. Thus, provided $a(t)$ is monotonic increasing with time, we find that for the viscoelastic solution meeting (13), (14)

$\sigma_{2 z}(x, 0, t)=\left(\frac{2}{\pi}\right)^{1 / 2}\left\{\frac{|x| g(a(t), t)}{\left[x^{2}-a^{2}(t)\right]^{1 / 2}}-g(0, t)-\int_{0}^{a(t)} \frac{|x| d g(v, t) / d v}{\left[x^{2}-v^{2}\right]^{1 / 2}} d v\right\}, \quad|x|>a(t)$.

1 The components of displacement and stress are independent of $y$ and, if we consider plane strain, $\sigma_{x y} \equiv \sigma_{z y} \equiv 0$. 
The conditions (13a, b), (14) and (18) together with the field equations (1)-(3) now determine a problem to which the classical correspondence principle is applicable. If $g(v, t)$ is differentiable in the neighborhood of $v=a$ we have that

$$
\int_{0}^{a} \frac{|x| d g(v, t) / d v}{\left[x^{2}-v^{2}\right]^{1 / 2}} d v=O(1) \quad \text { as } \quad x \rightarrow a^{+}(t) .
$$

so that the stress intensity factor, which is defined by the relation

$$
N(t)=\lim _{x \rightarrow a^{+}(t)}\left\{[x-a(t)]^{1 / 2} \sigma_{z z}(x, 0, t)\right\},
$$

is given by the relation

$$
N(t)=\left(\frac{a(t)}{\pi}\right)^{1 / 2} g(a(t), t)=\frac{(2 a(t))^{1 / 2}}{\pi} \int_{0}^{a(t)} \frac{p(|x|, t) d x}{\left[a^{2}(t)-x^{2}\right]^{1 / 2}} .
$$

It is also of interest to know the distribution of normal surface displacement over the region $|x| \leq a(t)$. By virtue of (24) and (25) of [5] and with the aid of (13), (16) we find that $^{2}$

$$
\begin{aligned}
u_{z}(x, 0, t)= & \left(\frac{2}{\pi}\right)^{1 / 2} K(0) H[a(t)-|x|] \int_{|x|}^{a(t)} \frac{v g(v, t)}{\left[v^{2}-x^{2}\right]^{1 / 2}} d v \\
& +\left(\frac{2}{\pi}\right)^{1 / 2} \int_{0}^{t} K^{\prime}(\theta)\left\{H[a(t-\theta)-|x|] \int_{|x|}^{o(t-\theta)} \frac{v g(v, t-\theta)}{\left[v^{2}-x^{2}\right]^{1 / 2}} d v\right\} d \theta,
\end{aligned}
$$

where

$$
K(t)=\mathcal{L}^{-1}\left[\frac{2\left[2 G_{1}^{T}(s)+G_{2}^{T}(s)\right]}{s^{2}\left[G_{1}^{T}(s)+2 G_{2}^{T}(s)\right] G_{1}^{T}(s)} ; s \rightarrow t\right] .
$$

If, in particular, $p$ is independent of $|x|$ so that $p(|x|, t)=p_{0}(t)$ then from (17) we find that

$$
g(v, t)=(\pi / 2)^{1 / 2} p_{0}(t) .
$$

Substituting from (24) into (18), (21) and (22) we find that

$$
\begin{gathered}
\sigma_{z z}(x, 0, t)=p_{0}(t)\left\{\frac{|x|}{\left[x^{2}-a^{2}(t)\right]^{1 / 2}}-1\right\}, \quad|x|>a(t), \\
N(t)=(a(t) / 2)^{1 / 2} p_{0}(t), \\
u_{z}(x, 0, t)=K(0) p_{0}(t) \operatorname{Re}\left\{\left[a^{2}(t)-x^{2}\right]^{1 / 2}\right\} \\
+\int_{0}^{t} K^{\prime}(\theta) p_{0}(t-\theta) \operatorname{Re}\left\{\left[a^{2}(t-\theta)-x^{2}\right]^{1 / 2}\right\} d \theta .
\end{gathered}
$$

If the viscoelastic material has similar behavior in shear and dilatation then we may find an unequivocal value of Poisson's ratio $\nu$ for it. It is easy to see that, in terms of $\nu, G_{1}$ and $G_{2}$ are then related through the equation

$$
G_{1}=\frac{1-2 v}{1+\nu} G_{2} .
$$

${ }^{2}$ Here and subsequently $H$ stands for the Heaviside unit step function which is defined by $H(\xi)=$ $0, \xi<0 ; H(\xi)=1,0 \leq \xi<\infty$.

${ }^{3}$ Here and subsequently "Re" indicates that we take the real part of the function shown. 
Substituting from (28) into (23) we see that

$$
K(t)=\mathscr{L}^{-1}\left[2(1-\nu) / s^{2} G_{1}^{T}(s) ; s \rightarrow t\right],
$$

which, with the aid of (11), implies that

$$
K(t)=2(1-\nu) G_{1}^{-1}(t) .
$$

For a Maxwell material whose behavior is characterized by

$$
G_{1}(t)=G_{0} e^{-t / \tau_{0}}, \quad G_{1}^{-1}(t)=\left(1 / G_{0}\right)\left(1+t / \tau_{0}\right)
$$

we find the aid of (27), (30) and (31) that ${ }^{4}$ :

$$
\begin{aligned}
& u_{\varepsilon}(x, 0, t) \\
& =\frac{2(1-\nu)}{G_{0}}\left\{p_{0}(t)\left[a^{2}(t)-x^{2}\right]^{1 / 2}+\frac{1}{\tau_{0}} \int_{0}^{t} p_{0}(\theta) \operatorname{Re}\left\{\left[a^{2}(\theta)-x^{2}\right]^{1 / 2}\right\} d \theta\right\}, \quad|x| \leq a(t) .
\end{aligned}
$$

In particular if $p_{0}$ is independent of time and $a$ is given by

$$
a(t)=v t,
$$

where $v$ is a constant, it is easy to verify that (32) reduces to

$$
\begin{aligned}
& u_{z}(x, 0, t)=\frac{2(1-\nu) p_{0}}{G_{0}}\left(v^{2} t^{2}-x^{2}\right)^{1 / 2} \\
& +\frac{2(1-\nu) p_{0}}{G_{0} \tau_{0}}\left\{\frac{|x| t}{2} \sinh \left[\cosh ^{-1}\left(\frac{v t}{|x|}\right)\right]-\frac{|x|^{2}}{2 v}\left[\cosh ^{-1}\left(\frac{v t}{|x|}\right)\right]\right\}, \quad|x| \leq v t .
\end{aligned}
$$

Normalized plots, at some distinct times, of $u_{z}(x, 0, t)$ as defined through (34) are given in Fig. 1 for $\tau_{0}=1, \nu=2$. (The plot corresponding to the case $t=10$ should, in fact, be drawn through the point with coordinates $(0,6)$.)

We will now consider the problem that arises when an infinite body containing a plane crack $z=0,|x| \leq a(t)$ is acted upon by certain stresses at infinity. Suppose that the boundary conditions and conditions at infinity take the form

$$
\begin{gathered}
\sigma_{z z}(x, 0, t)=\sigma_{z x}(x, 0, t)=0, \quad|x| \leq a(t), \\
\sigma_{z z}(x, z, t) \rightarrow p_{0}(t), \quad \sigma_{x x}(x, z, t) \rightarrow p_{1}(t), \quad \sigma_{z x}(x, z, t) \rightarrow 0, \quad \text { as } \quad\left(x^{2}+z^{2}\right) \rightarrow \infty .
\end{gathered}
$$

It is easy to verify that a solution to this problem is obtained by superimposing on the solution generated by (24) the following solution to the field equations (1)-(3):

$$
\begin{aligned}
& \sigma_{z z}=p_{0}(t), \quad \sigma_{x x}=p_{1}(t), \quad \sigma_{y y}=f(t), \quad \sigma_{z x}=\sigma_{z y}=\sigma_{x y}=0, \\
& \epsilon_{z z}=\frac{1}{3} p_{0} * d\left(G_{2}^{-1}+2 G_{1}^{-1}\right)+\frac{1}{3}\left(p_{1}+f\right) * d\left(G_{2}^{-1}-G_{1}^{-1}\right), \\
& \epsilon_{x x}=\frac{1}{3} p_{1} * d\left(G_{2}^{-1}+2 G_{1}^{-1}\right)+\frac{1}{3}\left(p_{0}+f\right) * d\left(G_{2}^{-1}-G_{1}^{-1}\right), \\
& \epsilon_{y y}=\frac{1}{3} f * d\left(G_{2}^{-1}+2 G_{1}^{-1}\right)+\frac{1}{3}\left(p_{0}+p_{1}\right) * d\left(G_{2}^{-1}-G_{1}^{-1}\right), \\
& \epsilon_{z x}=\epsilon_{z y}=\epsilon_{x y}=0, \\
& u_{z}=z \epsilon_{z z}, \quad u_{x}=x \epsilon_{x x}, \quad u_{y}=y \epsilon_{y y} .
\end{aligned}
$$

- A simple change of variable has been made in the integral occuring on the right-hand side of Eq. (32). 


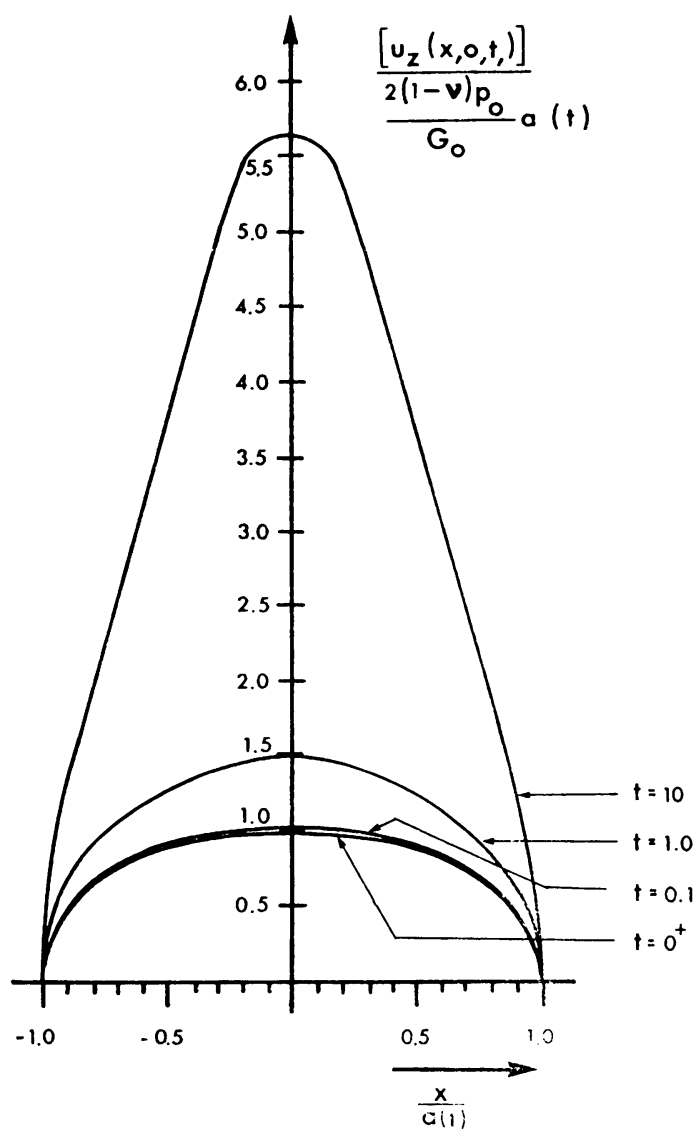

FIG. 1

By choosing

$$
f=\left(p_{0}+p_{1}\right) * d\left(G_{1}^{-1}-G_{2}^{-1}\right) * d\left(G_{2}^{-1}+2 G_{1}^{-1}\right)^{-1}
$$

we insure that (36) is a plane strain solution. By combining (25) with (36) it is found that, for the problem in hand,

$\sigma_{z z}[(a(t)+\epsilon), 0, t]=\sigma_{z z}[-(a(t)+\epsilon), 0, t]=N(t) / \sqrt{\epsilon}+O(\sqrt{\epsilon}) \quad$ as $\quad \epsilon \rightarrow 0$

where $N$ is given by (26). We also have that

$$
\sigma_{z x}(x, 0, t)=\sigma_{z y}(x, 0, t)=0, \quad-\infty<x<\infty .
$$

Let us suppose that, at some time $\tau$, the crack which has occupied the region $z=0$, $|x| \leq a(t)$ for times $t \leq \tau$, is given a smooth virtual extension so that at times $t, 0 \leq t \leq$ $\tau+\delta \tau$, it occupies the region $z=0,|x| \leq[a(t)+\delta(t)], 0 \leq t \leq \tau+\delta \tau$, where

$$
a(t)=a(\tau), \quad \tau \leq t \leq \tau+\delta \tau ; \quad \delta(t)=0, \quad 0 \leq t \leq \tau .
$$

The function $\delta(t), \tau<t<\tau+\delta \tau$, is assumed to be continuously differentiable with positive derivative. We suppose that as a result of this extension the field, which in the case that $\delta(t)=0, \tau<t<\tau+\delta \tau$ is given by $\left[u_{i}(t), \epsilon_{i j}(t), \sigma_{i j}(t)\right], 0 \leq t \leq \tau+\delta \tau$, is 
now given by $\left[u_{i}(t)+\delta u_{i}(t), \epsilon_{i j}(t)+\delta \epsilon_{i j}(t), \sigma_{i j}(t)+\delta \sigma_{i j}(t)\right], 0 \leq t \leq \tau+\delta \tau$, where $\left(\sigma_{z z}+\delta \sigma_{z z}\right)(x, 0, t)=\left(\sigma_{z x}+\delta \sigma_{z x}\right)(x, 0, t)=0, \quad|x| \leq[a(t)+\delta(t)]$,

$\left(\sigma_{z z}+\delta \sigma_{z z}\right)(x, 0, t) \rightarrow p_{0}(t), \quad\left(\sigma_{x x}+\delta \sigma_{x x}\right)(x, 0, t) \rightarrow p_{1}(t)$,

$$
\left(\sigma_{z x}+\delta \sigma_{z x}\right)(x, 0, t) \rightarrow 0 \quad \text { as } \quad\left(x^{2}+z^{2}\right) \rightarrow \infty, \quad 0 \leq t \leq \tau \leq \delta \tau .
$$

With this notation we have, by virtue of the second part of (40), that

$$
\delta u_{i}(t) \equiv \delta \epsilon_{i j}(t) \equiv \delta \sigma_{i j}(t) \equiv 0, \quad 0 \leq t \leq \tau .
$$

With the aid of (27), (36) and (40) we find that ${ }^{5}$

$$
\begin{aligned}
\delta u_{z}(x, 0, t)= & K(0) p_{0}(t) \operatorname{Re}\left\{[a(t)+\delta(t)]^{2}-x^{2}\right\}^{1 / 2} \\
& +\int_{0}^{t-\tau} K^{\prime}(\theta) p_{0}(t-\theta) \operatorname{Re}\left\{[a(t-\theta)+\delta(t-\theta)]^{2}-x^{2}\right\}^{1 / 2} d \theta, \\
& a(\tau) \leq x \leq a(\tau)+\delta(\tau+\delta \tau), \quad \tau \leq t \leq \tau+\delta \tau .
\end{aligned}
$$

By combining (26) with (43) we find that

$$
\begin{aligned}
\delta u_{z}\{[a(t)+\epsilon], 0, t\} & =\delta u_{z}\{-[a(t)+\epsilon], 0, t\} \\
& =2 K(0) N(t) \sqrt{[\delta(t)-\epsilon]}+O\left\{(\delta(t))^{3 / 2}\right\}, \quad \text { as } \delta(t) \rightarrow 0^{+} .
\end{aligned}
$$

In line with (39) we have that

$$
\delta \sigma_{z x}(x, 0, t)=\delta \sigma_{z y}(x, 0, t)=0, \quad-\infty<x<\infty, \tau \leq t \leq \tau+\delta \tau .
$$

We will denote by $\varepsilon(t)$ the increment of work performed in extending the semidiameter of a crack by an amount $\delta(t), \tau \leq t \leq \tau+\delta \tau$. Then we find that

$$
\begin{array}{r}
\frac{d \varepsilon}{d t}=\int_{B} T_{i}(t) \frac{d}{d t}\left(\delta u_{i}(t)\right) d A+\int_{B} \delta T_{i}(t) \frac{d}{d t}\left(\delta u_{i}(t)\right) d A+\int_{B} \delta T_{i}(t) \frac{d}{d t}\left(u_{i}(t)\right) d A, \\
\tau<t<\tau+\delta \tau,
\end{array}
$$

where $T_{i}(t)=\sigma_{i j}(t) n_{i}$ and $\delta T_{i}(t)=\delta \sigma_{i j}(t) n_{i}$ while $B$ denotes the boundary of the body after the extension of the crack semidiameter by amount $\delta(\tau+\delta \tau)$ and $n_{i}$ are the components of the outward unit normal to $B$. With the aid of (35), (41), (45) together with the first part of (40) and (27) it may be checked that the third term in (46) vanishes ${ }^{6}$. Further, by using the divergence theorem and the stress-strain relationship (3), (4) along with (42) we find from (46) that

$$
\left.\frac{d \varepsilon}{d t}\right|_{t=\tau^{+}}=\left.\int_{B} T_{i}(\tau) \frac{d}{d t}\left(\delta u_{i}(t)\right)\right|_{t=\tau^{+}} d A+\left.\frac{1}{2} \frac{d}{d t} \int_{B} \delta T_{i}(t) \delta u_{i}(t) d A\right|_{t=\tau^{+}},
$$

where we have put to zero, when $t=\tau^{+}$, certain hereditary integrals over the range $[0, t-\tau]$. Now, at each point of the boundary $B$, either $T_{i}(\tau)$ is prescribed or alternatively it has the same value as that which would be obtained from an elastic analysis based on

${ }^{5}$ In deriving (43) and (45) we use the fact that the solution we have obtained to the problem governed by (35) is valid for monotone increasing $a(t)$.

6 With the aid of $(36)$ it is seen that the value of $u_{z}(x, 0, t)$ given by (27) is valid for the viscoelastic problem governed by (35). 
the elastic constants $\mu=\frac{1}{2} G_{1}(0), \kappa=\frac{1}{3} G_{2}(0)$ and zero body force ${ }^{7}$. Further, by using (42), we find from an elementary extension of Theorem (6.2) of Gurtin and Sternberg [9] that

$$
\left\{\left.\frac{d}{d t}\left[\delta u_{i}(t)\right]\right|_{t=\tau^{+}},\left.\frac{d}{d t}\left[\delta \epsilon_{i i}(t)\right]\right|_{t=\tau^{+}},\left.\frac{d}{d t}\left[\delta \sigma_{i j}(t)\right]\right|_{t=\tau^{+}}\right\}
$$

is an elastic state corresponding to the same elastic constants and zero body force. Thus, by using Bettis theorem (see [10]) we find that

$$
\left.\int_{B} T_{i}(\tau) \frac{d}{d t}\left[\delta u_{i}(t)\right]\right|_{t=\tau^{+}} d A=\left.\int_{B} u_{i}^{\prime}(\tau) \frac{d}{d t}\left[\delta T_{i}(t)\right]\right|_{t=\tau^{+}} d A,
$$

where $u_{i}^{\prime}$ stands for the values of the displacements obtained from an elastic analysis to the crack problem in hand, with elastic constants $\mu=\frac{1}{2} G_{1}(0), \kappa=\frac{1}{3} G_{2}(0)$. By virtue of (48) and with the aid of (35), (41), (45) and the form of (27) obtained by taking $K^{\prime} \equiv 0$ it is seen that the first term of (47) vanishes. At the same time by using (43), we find that

$$
\left.\frac{d \varepsilon}{d t}\right|_{t=\tau^{+}}=-\left.2 \frac{d}{d t} \int_{0}^{\delta(r)} \sigma_{z z}[(a(t)+\epsilon), 0, t] \delta u_{z}[(a(t)+\epsilon), 0, t] d \epsilon\right|_{t=\tau^{+}} .
$$

On substituting from (38) and (44) in (49) and taking account of (26) and (40), we find that $^{9}$

$$
\left.\frac{d \varepsilon}{d t}\right|_{t=\tau^{+}}=-\left.\pi K(0) a(\tau) p_{0}^{2}(\tau) \frac{d \delta}{d t}\right|_{t=\tau^{+}} .
$$

We will now assume that if the crack extends in overall length by an amount $2 \delta$ then an amount of work $4 T \delta$ must be done where $T$ is an energy characteristic of the material ${ }^{10}$. Then, following Griffith [6], a necessary condition for crack growth is that the rate of liberation of energy due to crack extension should not be less than the rate of doing work in crack tearing. Thus a condition for the extension of the crack is found to be that

$$
a(\tau) p_{0}^{2}(\tau) \geq 4 T / \pi K(0)
$$

which is the same as the condition that would be obtained from an elastic analysis based on the initial values of the relaxation moduli and the current values of the applied load and size of crack (see Sneddon [7]). On using (26) we find that (51) may be written in the form

$$
N^{2}(\tau) \geq 2 T / \pi K(0)
$$

4. A three-dimensional extending crack problem. In this section we first give the solution to the problem of a plane circular extending crack in an infinite linear viscoelastic material. In terms of circular cylindrical coordinates $(\rho, \varphi, z)$ the distribution of

${ }^{7} \mu$ stands for shear modulus and $\kappa$ for bulk modulus.

${ }^{8}$ Cf. footnote 6.

${ }^{9}$ Here we have used the fact that in (38) and (44) the symbol $\mathrm{O}\left(\delta^{3 / 2}\right)$, for example, is used to designate a quantity which has, in fact, the same order of magnitude as $\delta^{3 / 2}$. This is not quite the usual mathematical meaning of $\mathrm{O}(\mathrm{)}$.

${ }_{10}$ The existance of such characteristic energies of tearing has been demonstrated for certain rubber vulcanizates by Rivlin and Thomas [11]. 
stress and displacement is the same as that in a semi-infinite body when its surface is subject to the boundary conditions ${ }^{11}$

$$
\begin{aligned}
& \sigma_{z \rho}(\rho, 0, t)=\sigma_{z \varphi}(\rho, 0, t)=0, \quad \rho \geq 0, \\
& \sigma_{z z}(\rho, 0, t)=-p(\rho, t), \quad 0 \leq \rho \leq a(t), \\
& u_{z}(\rho, 0, t)=0, \quad \rho>a(t),
\end{aligned}
$$

and the conditions at infinity

$$
\sigma_{i j}(\rho, z, t) \rightarrow 0 \text { as }\left(\rho^{2}+z^{2}\right) \rightarrow \infty, \text { all } i, j .
$$

Here $a(t)$, which gives the radius of the circular crack at time $t$, is assumed to be a monotonic increasing function of time. The function $p(\rho, t)$ gives the distribution of pressure that acts over the surface of the crack. Denoting by the superscript $e$ the one-parameter family of solutions determined by the conditions (53), (54) and the field equations (1), (2) and (12), we have in particular that (see Sneddon [12])

$$
\begin{gathered}
\sigma_{z z}^{e}(\rho, 0, t)=\frac{g(a(t), t)}{\left[\rho^{2}-a^{2}(t)\right]^{1 / 2}}-\int_{0}^{a(t)} \frac{\partial g(v, t) / \partial v}{\left[\rho^{2}-v^{2}\right]} d v, \quad \rho>a(t), \\
u_{z}^{e}(\rho, 0, t)=\frac{1-\nu}{\mu} \int_{\rho}^{a(t)} \frac{g(v, t)}{\left[v^{2}-\rho^{2}\right]^{1 / 2}} d v, \quad 0 \leq \rho \leq a(t),
\end{gathered}
$$

where

$$
g(v, t)=\frac{2}{\pi} \int_{0}^{v} \frac{\rho p(\rho, t) d \rho}{\left[v^{2}-\rho^{2}\right]^{1 / 2}}
$$

From the form taken by (55) and (56) we see that the problem in hand is one to which the extended correspondence principle [5] is applicable. We therefore have that

$$
\sigma_{z z}(\rho, 0, t)=\frac{g(a(t), t)}{\left[\rho^{2}-a^{2}(t)\right]^{1 / 2}}-\int_{0}^{a(t)} \frac{\partial g(v, t) / \partial v}{\left[\rho^{2}-v^{2}\right]^{1 / 2}} d v, \quad \rho>a(t) .
$$

In particular, if $g(v, t)$ is differentiable in the neighborhood of $v=a$ we find that the stress intensity factor, which is defined through

$$
N(t)=\lim _{\rho \rightarrow a^{+}(t)}\left\{[\rho-a(t)]^{1 / 2} \sigma_{z 2}(\rho, 0, t)\right\},
$$

is given by

$$
N(t)=\frac{g(a(t), t)}{[2 a(t)]^{1 / 2}}=\frac{1}{\pi}\left(\frac{2}{a(t)}\right)^{1 / 2} \int_{0}^{a(t)} \frac{\rho p(\rho, t) d \rho}{\left[a^{2}(t)-\rho^{2}\right]^{1 / 2}} .
$$

In a manner similar to that used in the previous section we find that

$$
\begin{aligned}
u_{z}(\rho, 0, t)=K(0) H[a(t) & -\rho] \int_{\rho}^{a(t)} \frac{g(v, t) d v}{\left[v^{2}-\rho^{2}\right]^{1 / 2}} \\
& +\int_{0}^{t} K^{\prime}(\theta)\left\{H(a[t-\theta]-\rho) \int_{\rho}^{a(t-\theta)} \frac{g(v, t-\theta)}{\left[v^{2}-\rho^{2}\right]^{1 / 2}} d v\right\} d \theta,
\end{aligned}
$$

where the auxiliary response function $K$ is given by (23).

11 The components of displacement and stress are independent of $\varphi$. 
If $p$ is independent of $\rho$ and is given by $p(\rho, t)=p_{0}(t)$ then from (57) we find that

$$
g(v, t)=(2 / \pi) p_{0}(t)|v| \text {. }
$$

By substituting from (62) into (58), (60) and (61) we find that

$$
\begin{aligned}
\sigma_{s z}(\rho, 0, t) & =\frac{2}{\pi} p_{0}(t)\left[\frac{a(t)}{\left[\rho^{2}-a^{2}(t)\right]^{1 / 2}}-\sin ^{-1}\left(\frac{a(t)}{\rho}\right)\right], \quad \rho>a(t), \\
N(t) & =\frac{\sqrt{2}}{\pi} p_{0}(t)[a(t)]^{1 / 2}, \\
u_{3}(\rho, 0, t)= & \frac{2}{\pi} K(0) p_{0}(t) \operatorname{Re}\left\{\left[a^{2}(t)-\rho^{2}\right]^{1 / 2}\right\} \\
& \quad+\frac{2}{\pi} \int_{0}^{t} K^{\prime}(\theta) p_{0}(t-\theta) \operatorname{Re}\left\{\left[a^{2}(t-\theta)-\rho^{2}\right]^{1 / 2}\right\} d \theta .
\end{aligned}
$$

We will now briefly consider the problem that arises when an infinite viscoelastic body contains a circular crack $z=0, \rho \leq a(t)$ which is opened up by the action of stresses at infinity. Consider the viscoelastic problem governed by the conditions

$\sigma_{z z}(\rho, 0, t)=\sigma_{z \rho}(\rho, 0, t)=\sigma_{z \varphi}(\rho, 0, t)=0, \quad \rho \leq a(t)$,

$\sigma_{z z}(\rho, z, t) \rightarrow p_{0}(t), \quad \sigma_{\rho \rho}(\rho, z, t) \rightarrow p_{1}(t), \quad \sigma_{\varphi \varphi}(\rho, z, t) \rightarrow p_{1}(t) \quad$ as $\quad\left(\rho^{2}+z^{2}\right) \rightarrow \infty$,

$\sigma_{z \rho}(\rho, z, t) \rightarrow 0, \quad \sigma_{z \varphi}(\rho, z, t) \rightarrow 0, \quad \sigma_{\rho \varphi}(\rho, z, t) \rightarrow 0 \quad$ as $\quad\left(\rho^{2}+z^{2}\right) \rightarrow \infty$.

It is easy to verify that a solution to this problem is obtained by superimposing on the solution generated by (62) the following solution to the field equations (1)-(3):

$$
\begin{aligned}
& \sigma_{z z}=p_{0}(t), \quad \sigma_{\rho \rho}=\sigma_{\varphi \varphi}=p_{1}(t), \quad \sigma_{z \rho}=\sigma_{z \varphi}=\sigma_{\rho \varphi}=0, \\
& \epsilon_{z z}=\frac{1}{3} p_{0} * d\left(G_{2}^{-1}+2 G_{1}^{-1}\right)+\frac{2}{3} p_{1} * d\left(G_{2}^{-1}-G_{1}^{-1}\right), \\
& \epsilon_{\rho \rho}=\epsilon_{\varphi \varphi}=\frac{1}{3} p_{0} * d\left(G_{2}^{-1}-G_{1}^{-1}\right)+\frac{1}{3} p_{1} * d\left(2 G_{2}^{-1}+G_{1}^{-1}\right), \\
& \epsilon_{z \rho}=\epsilon_{z \varphi}=\epsilon_{\rho \varphi}=0, \\
& u_{z}=z \epsilon_{z z}, \quad u_{\rho}=\rho \epsilon_{\rho \rho}, \quad u_{\varphi}=0 .
\end{aligned}
$$

By combining (63) with (67) it is found that, for the problem now at hand,

$$
\sigma_{z z}[(a(t)+\epsilon), 0, t]=N(t) / \sqrt{\epsilon}+O(\sqrt{\epsilon}) \quad \text { as } \epsilon \rightarrow 0^{+},
$$

where $N$ is given by (64). We also have that

$$
\sigma_{z \rho}(\rho, 0, t)=\sigma_{z \varphi}(\rho, 0, t)=0, \quad \rho>0 .
$$

Suppose that, at time $\tau$, the crack which has occupied the region $z=0, \rho \leq a(t)$ for times $t \leq \tau$ is given a smooth virtual extension so that at times $t, 0 \leq t \leq \tau+\delta \tau$, it occupies the region $z=0, \rho \leq[a(t)+\delta(t)], 0 \leq t \leq \tau+\delta \tau$, where the functions $a$ and $\delta$ have the same properties as in the previous section. The field $\left[u_{i}(t), \epsilon_{i j}(t), \sigma_{i j}(t)\right]$ is then replaced by $\left[u_{i}(t)+\delta u_{i}(t), \epsilon_{i j}(t)+\delta \epsilon_{i j}(t), \sigma_{i j}(t)+\delta \sigma_{i j}(t)\right]$ which, in analogy with (41) in the previous section, satisfies the conditions (66) with $a(t)$ replaced by $a(t)+\delta(t)$, $0 \leq t \leq \tau+\delta \tau$. The conditions (42) are clearly met and by virtue of (65), (67) and (64) we find, as before, that

$$
\delta u_{\mathbf{s}}\{[a(t)+\epsilon], 0, t\}=2 K(0) N(t) \sqrt{[\delta(t)-\epsilon]}+O\left\{[\delta(t)]^{3 / 2}\right\}, \quad \text { as } \quad \delta(t) \rightarrow 0^{+},
$$


while (69) gives us that

$$
\delta \sigma_{z \rho}(\rho, 0, t)=\delta \sigma_{z_{\varphi}}(\rho, 0, t)=0, \quad \rho>0 .
$$

Then, using the same arguments as before, we find that

$$
\left.\frac{d \mathcal{E}}{d t}\right|_{t=\tau^{+}}=-\left.\pi \frac{d}{d t} \int_{0}^{\delta(\tau)}(a(t)+\epsilon) \sigma_{z z}[(a(t)+\epsilon), 0, t] \delta u_{z}[(a(t)+\epsilon), 0, t] d \epsilon\right|_{t=\tau^{+}} \text {, }
$$

which, on substituting from (68) and (70), and using (64) and (40) gives us, in the same way that we obtained (50), that

$$
\left.\frac{d \varepsilon}{d t}\right|_{t=\tau^{+}}=-\left.2 K(0) p_{0}^{2}(\tau) a^{2}(\tau) \frac{d \delta}{d t}\right|_{t=\tau^{+}}
$$

Now if we assume that in extending the radius of the crack by $\delta$ an amount of work $\pi T\left[(a(\tau)+\delta)^{2}-a^{2}(\tau)\right]$ must be done, then we find that a condition for the growth of a circular crack is given by

$$
a(\tau) p_{0}^{2}(\tau) \geq \pi T / K(0) .
$$

On substituting from (64) we find that this condition may be written in the form (52). The condition (74) is the same as that which may be obtained from an elastic analysis based on the initial values of the relaxation moduli (see Sneddon [7]).

We will now briefly discuss the results embodied in (51) and (74). Both these conditions involve the time as a parameter only. They divide the quarter plane of points with rectangular Cartesian coordinates $(a, p)$ into a "stable part" and an "unstable part". The crack will not extend as long as $(a, p)$ is in the stable part; however, at the first instance that $(a, p)$ enters the unstable part, the crack should extend without limit.

\section{REFERENCES}

1. E. H. Lee, Stress analysis in visco-elastic bodies, Quart. Appl. Math. (2) 13, 183-190 (1955)

2. M. L. Williams, P. J. Blataz and R. A. Schapery, Fundamental studies relating to systems analysis of solid propellants, Guggenheim Aeronautical Laboratory, California Institute of Technology, Pasadena, Calif., 1961

3. M. L. Williams, Initiation and growth of viscoelastic fracture, Internat. J. Fracture Mechanics (4) 1, 292-310 (1965)

4. J. R. Willis, Crack propagation in viscoelastic media, J. Mech. Phys. Solids (4) 15, 229-240 (1967)

5. G. A. C. Graham, The correspondence principle of linear viscoelasticity theory for mixed boundary value problems involving time-dependent boundary regions, Quart. Appl. Math. (2) 26, 167-174 (1968)

6. A. A. Griffith, The theory of rupture, Proc. 1st Internat. Congress Appl. Mech. Delft, 55-63 (1924)

7. I. N. Sneddon, The distribution of stress in the neighborhood of a crack in an elastic solid, Proc. Royal Soc. Ser. A187, 229-260 (1946)

8. I. N. Sneddon, The use of transform methods in elasticity, Applied Mathematics Research Group, North Carolina State University, Raleigh, N. C., 1964

9. M. E. Gurtin and E. Sternberg, On the linear theory of viscoelasticity, Arch. Rational Mech. Anal. (4) $11,291-356(1962)$

10. I. S. Sokolnikoff, Mathematical theory of elasticity, McGraw-Hill, New York, 1956

11. R. S. Rivlin and A. G. Thomas, Rupture of rubber. I: Characteristic energy for tearing, J. Polymer Sci. (3) 10, 291-318 (1953)

12. I. N. Sneddon, $A$ note on the problem of the penny shaped crack, Proc. Cambridge Philos. Soc. 61 609-611 (1965) 